\title{
RENAL INJURY AND DYSFUNCTION AMONG HIV POSITIVE PATIENTS RECEIVING TENOFOVIR BASED ANTI-RETROVIRAL THERAPY
}

Wasin Bunpeth, Ouppatham Supasyndh, Bancha Satirapoj

Division of Nephrology, Department of Medicine, Phramongkutklao College of Medicine , Bangkok, Thailand

\begin{abstract}
The rate of renal disease among patients with HIV has decreased significantly since the introduction of highly active antiretroviral therapy (HAART). Patients receiving tenofovir, disoproxil, fumarate (TDF) had an increased prevalence of proximal renal tubular dysfunction and injury but its clinical significance remain controversial.To define the renal tubulopathy injury among patients with HIV with and without TDF. A cross-sectional study was conducted among HIV positive patients receiving TDF $(\mathrm{N}=176)$ and nonTDF regimen $(\mathrm{N}=146)$ at outpatient clinic. All patients were evaluated regarding serum creatinine, electrolytes, phosphate and differing urinary parameters (proteinuria, glycosuria and pyuria). Estimated glomerular filtration rate (GFR) was calculated using CKD-EPI equation. Of 322 participants with mean age of $41.6 \pm 11.4$ years and HIV duration of $7.2 \pm 4.3$ years, the TDF and nonTDF groups were similar on most clinical and demographic factors. GFR was $100.6 \pm 17.8 \mathrm{~mL} / \mathrm{min} / 1.73 \mathrm{~m}{ }^{2}$ in TDF group and $97.5 \pm 19.6 \mathrm{~mL} / \mathrm{min} / 1.73 \mathrm{~m}^{2}$ in non-TDF group $(p=0.143)$. During evaluation, $3.4 \%$ of TDF patients vs. none of the nonTDF - patients had hypophosphataemia $(<2.5 \mathrm{mg} / \mathrm{dL}), 3.9 \%$ of TDF - patients vs. $1.3 \%$ of nonTDF had hypokalemia ( $<3.5 \mathrm{mg} / \mathrm{dL})$, and $0.68 \%$ of TDF - patients vs. none of nonTDF patients had acidosis $(<18 \mathrm{mEq} / \mathrm{L})$ with no statistically significant difference between groups. The proportion of patients with evidence of urine abnormalities was also similar in the two groups (Dipstick proteinuria $>1+$; TDF: $17.6 \%$ vs. non-TDF $20.5 \%, p=0.568$, and pyuria; TDF: $27.3 \%$ vs. nonTDF $20.5 \%, p=0.192$ ). Renal impairment, electrolyte disturbances and renal tubulopathy were uncommon among HIV positive patients receiving TDF-based antiretroviral therapy and did not significantly differ between TDF and nonTDF regimens.
\end{abstract}

Keywords : Tenofovir, Tubulopathy, Acute kidney injury

J Southeast Asian Med Res 2017; 1: 6-11.

http://www.jseamed.org

\section{Correspondence to:}

Satirapoj B, Division of Nephrology, Department of Medicine, Phramongkutklao College of Medicine, Bangkok, Thailand

E-mail : satirapoj@yahoo.com 


\section{Introduction}

Highly active antiretroviral therapy (HAART) has reduced the mortality and morbidity associated with human immunodeficiency virus (HIV) infection. Tenofovir disoproxil fumarate, a prodrug of tenofovir (TDF), is a potent nucleotide analogue reverse transcriptase inhibitor and the first line HAART regimens according to a recommendation of the World Health Organization. TDF is mainly elimi- nated by glomerular filtration and $20 \%$ to $30 \%$ by proximal tubular secretion. Initially, case reports demonstrated TDF - associated acute tubular necrosis, proximal tubular injury and Fanconi syndrome with hypouricemia and hypophosphatemia. ${ }^{(1,2)}$ In a cohort study, TDF based anti-retroviral therapywas associated with GFR decline during the first years of treatment and relatively mild GFR decline in a long-term follow-up. ${ }^{(3)}$

Currently, TDF based antiretroviral therapy has been reported infrequently in renal toxicity and renal tubular dysfunction among of HIV positive patients. ${ }^{(4,5)}$ The high incidence of TDF-associated nephropathy is related to aging, low body weight, low CD4 cell count, advanced HIV disease, preexisting kidney disease, concomitant hepatitis $\mathrm{C}$ virus ( $\mathrm{HCV}$ ) infection and concurrent use of other nephrotoxic drugs. ${ }^{(6-9)}$ Little is known about renal safety of TDF among Thai patients on TDF. Here we report the results assessing renal safety of TDF and nonTDF based antiretro- viral therapy among Thai HIV positive patients.

\section{Methods}

Study design and population

The cross-sectional study evaluated the renal safety of TDF treatment among HIV positive patients during January 2014 and December 2014. The study protocol was approved by the institutional review boards and ethics committees. Written informed consent was obtained from all patients at screening. The primary objective was to determine the renal function and renal tubular defects between HIV positive patient treatment with TDF and non TDF regimens. Eligibility included male and female patients aged 18 to 85 years diagnosed with HIV receiving antiretroviral therapy treatment at least 12 weeks.

\section{Measurements}

The clinical and laboratory data were collected and recorded on case report forms. Safety was assessed by physical examinations, clinical laboratory tests and the incidence and severity of adverse events recorded from treatment. Serum creatinine, electrolytes, calcium, phosphate, uric acid, CD4 + cell count, plasma HIV-1 viral load and urine analysis were measured. The estimated glomerular filtration rate (GFR) was calculated using the CKD-EPI equation.

Urine protein was measured by a urine dipstick. Albuminuria and glycosuria were defined as $\geq 1+$ on a urine dipstick. Pyuria was defined as $\geq 3$ white blood cells per high power field urinalysis.

Statistical analysis

Data were expressed as mean \pm standard deviation (SD) and median with interquartile range. Continuous variables were assessed with the Student's t-test or Mann-Whitney U test appropriately. Categorical variables were assessed with the Chi-square test. For all tests, a $p$-value less than 0.05 was considered statistically significant. Statistical analysis was conducted using SPSS version 16.0 (SPSS Inc., Chicago, IL, USA). Statistical significance was determined as a $p$ - value less than 0.05 .

\section{Results}

A total of 322 ( $100 \%$ Thai ethnicity) with a mean age of $41.6 \pm 11.4$ years and HIV duration of $7.2 \pm 4.3$ years were included. Baseline demographics of the TDF and nonTDF patients are shown in Table 1. Compared with the nonTDF group, the TDF group exhibited lower mean age, CD4 count and the comorbid diseases of hypertension and dyslipidemia.The duration of HARRT treatment was significantly longer in the nonTDF group compared with the TDF group. No significant differences in other baseline characteristics were noted between the two groups Table 1 .Common medications and antiretroviral therapy among TDF and nonTDF patients are shown in Table 2. Mean GFR was $100.6 \pm 17.8 \mathrm{~mL} / \mathrm{min} / 1.73 \mathrm{~m}^{2}$ in the TDF group and $97.5 \pm 19.6 \mathrm{~mL} / \mathrm{min} / 1.73 \mathrm{~m}^{2}$ in the nonTDF group $(p=0.143)$. 
Both serum creatinine and estimated GFR were similar across treatment groups Table 3. Overall tubular dysfunction levels regarding serum electrolytes and urine findings are shown in Fig 1. No significant difference in impaired GFR, hypophosphataemia, hypokalemia and metabolic acidosis incidence between the two groups was apparent. The proportion of patients with evidence of urine abnorma- lities was also similar in the two groups (Dipstick urine albumin $>1+$; TDF: $17.6 \%$ vs. nonTDF $20.5 \%, p=$ 0.568 , dipstick urine glucose $>1+$; TDF: $17.6 \%$ vs. nonTDF $20.5 \%, p=0.568$ and pyuria; TDF: $27.3 \%$ vs. nonTDF $20.5 \%, p=0.192)$. Finally, no clear difference in all renal functions and biochemical tubular parameters across treatment in either study was detected. Overall serious renal adverse events were not reported in both group.

Table 1. Baseline characteristics

\begin{tabular}{|c|c|c|c|}
\hline & TDF & Non-TDF & $p$-value \\
\hline & $(\mathrm{N}=176)$ & $(\mathrm{N}=146)$ & \\
\hline Age (yr) & $38.4 \pm 11.5$ & $45.3 \pm 10.0$ & 0.000 \\
\hline Male (N, \%) & $127(72.2 \%)$ & $96(65.8 \%)$ & 0.227 \\
\hline Blody weight (kg) & $61.4 \pm 12.2$ & $61.1 \pm 10.5$ & 0.775 \\
\hline Body mass index $\left(\mathrm{kg} / \mathrm{m}^{2}\right)$ & $22.0 \pm 3.9$ & $22.4 \pm 3.5$ & 0.395 \\
\hline Systolic blood pressure ( $\mathrm{mmHg}$ ) & $123.8 \pm 14.2$ & $126.3 \pm 16.4$ & 0.152 \\
\hline Diastolic blood pressure ( $\mathrm{mmHg}$ ) & $77.0 \pm 11,4$ & $78.8 \pm 12,1$ & 0.192 \\
\hline Duration of antiretrowiral therapy $(\mathrm{yr})$ & $5.4 \pm 4.0$ & $9.5 \pm 3.5$ & 0.000 \\
\hline \multicolumn{4}{|l|}{ Co-morbid diseases $(\mathbb{N}, \%)$} \\
\hline - Hypertension & $14(8 \%)$ & $39(26.7 \%)$ & 0.000 \\
\hline - Dyslipidemia & $32(18.2 \%)$ & $76(52.1 \%)$ & 0.000 \\
\hline - Type 2 diabetes & $5(2.8 \%)$ & $9(6.2 \%)$ & 0.175 \\
\hline - Ischemie heart disease & $9(7.9 \%)$ & $12(12.1 \%)$ & 0.333 \\
\hline \multicolumn{4}{|l|}{ Common co-infection (N, \%) } \\
\hline -Mycobacterium tuberculosis & $59(33.5 \%)$ & $49(33.6 \%)$ & 1.000 \\
\hline -Pneumacystis jirovedi & $33(18.8 \%)$ & $23(15.8 \%)$ & 0.555 \\
\hline - Cryptococcus neofarmans & $13(7,4 \%)$ & $11(7.5 \%)$ & 1.000 \\
\hline -Treponema pallidum & $12(6.8 \%)$ & $6(4.1 \%)$ & 0.338 \\
\hline - Candida albicans & $12(6.8 \%)$ & $4(2.7 \%)$ & 0.123 \\
\hline CD4 count (cell/mm') & $437.2 \pm 227.1$ & $543.8 \pm 234.5$ & 0.000 \\
\hline HIV RNA viral load (copies/mL) & $560+3226$ & $1509.6 \pm 16365.2$ & 0.505 \\
\hline
\end{tabular}

Data are expressed as mean SD, median (interquartile) or as number (percentage) of patients. Comparisons between treatment groups employed the Independent $t$-test (continuous variables) and Chi-square test (Categorical variables).
Table 2. Medical treatments among HIV positive patients receiving TDF and nonTDF regimens

\begin{tabular}{|c|c|c|c|}
\hline & $\begin{array}{c}\text { TDF } \\
(\mathrm{N}=176)\end{array}$ & $\begin{array}{l}\text { Non-TDF } \\
(N=146)\end{array}$ & $p$-value \\
\hline \multicolumn{4}{|c|}{ Antiretroviral medications $(\mathrm{N}, \%)$} \\
\hline Zidovudine (AZT) & $9(5.1 \%)$ & $95(65.1 \%)$ & 0.000 \\
\hline Lamivudine (3TC) & $165(93.8 \%)$ & $114(78.1 \%)$ & 0.000 \\
\hline Nevirapine (NVP) & $19(10.8 \%)$ & $40(27.4 \%)$ & 0.000 \\
\hline Efavirenz (EFV) & $123(69.9 \%)$ & $52(35.6 \%)$ & 0.000 \\
\hline Stavudine (D4T) & $1(0.6 \%)$ & $19(13 \%)$ & 0.000 \\
\hline Didanosine (DD) & $1(0.6 \%)$ & $3(2.1 \%)$ & 0.333 \\
\hline Lopinavir (LPV) & $28(15.9 \%)$ & $24(16.4 \%)$ & 1.000 \\
\hline Atazanavir (ATV) & $6(3,4 \%)$ & $4(2.7 \%)$ & 1.000 \\
\hline \multicolumn{4}{|c|}{ Other medientions ( $\mathrm{N}, \%)$} \\
\hline Statin & $13(7.4 \%)$ & $44(30.1 \%)$ & 0.000 \\
\hline Fibrate & $20(11.4 \%)$ & $33(22.6 \%)$ & 0.010 \\
\hline Co-trimoxazole & $18(10.2 \%)$ & $2(2.1 \%)$ & 0.003 \\
\hline Fluconazole & $17(9.7 \%)$ & $1(0.7 \%)$ & 0.000 \\
\hline
\end{tabular}

Data are expressed as number (percentage) of patients. Comparisons between treatment groups employed the Chi-square test (Categorical variables).

Table 3. Renal function and serum electrolytes among HIV positive patients receiving TDF and nonTDF regimens

\begin{tabular}{lccc}
\hline & TDF & Non-TDF & $p$-value \\
& $(\mathrm{N}=176)$ & $(\mathrm{N}=146)$ & \\
\hline BUN (mg/dL) & $10.9 \pm 4.2$ & $12 \pm 6.9$ & 0.091 \\
Serum Cr (mg/dL) & $0.9 \pm 0.2$ & $0.91 \pm 0.6$ & 0.842 \\
Estimated GFR (mL/min/l.73 m ) & $100.7 \pm 17.8$ & $97.5 \pm 19.6$ & 0.134 \\
$\mathrm{Na}(\mathrm{mEq} / \mathrm{L})$ & $139 \pm 2.1$ & $139 \pm 1.9$ & 0.432 \\
$\mathrm{~K}(\mathrm{mEq} / \mathrm{L})$ & $4.1 \pm 0.4$ & $4.1 \pm 0.3$ & 0.329 \\
$\mathrm{Cl}(\mathrm{mEq} / \mathrm{L})$ & $102 \pm 2.5$ & $102 \pm 2.2$ & 0.101 \\
$\mathrm{HCO}(\mathrm{mEq} / \mathrm{L})$ & $26.2 \pm 2.6$ & $25.85 \pm 2.5$ & 0.225 \\
$\mathrm{Ca}(\mathrm{mg} / \mathrm{dL})$ & $9.2 \pm 0.4$ & $9.2 \pm 0.5$ & 0.415 \\
$\mathrm{PO} 4(\mathrm{mg} / \mathrm{dL})$ & $3.2 \pm 0.5$ & $3.4 \pm 0.5$ & 0.160 \\
$\mathrm{U}$ Uric (mg/dL) & $5.1 \pm 1.5$ & $5.2 \pm 1.6$ & 0.565 \\
\hline
\end{tabular}

Data are expressed as mean SD. Comparisons between treatment groups using the Independent $t$-test (continuous variables). 


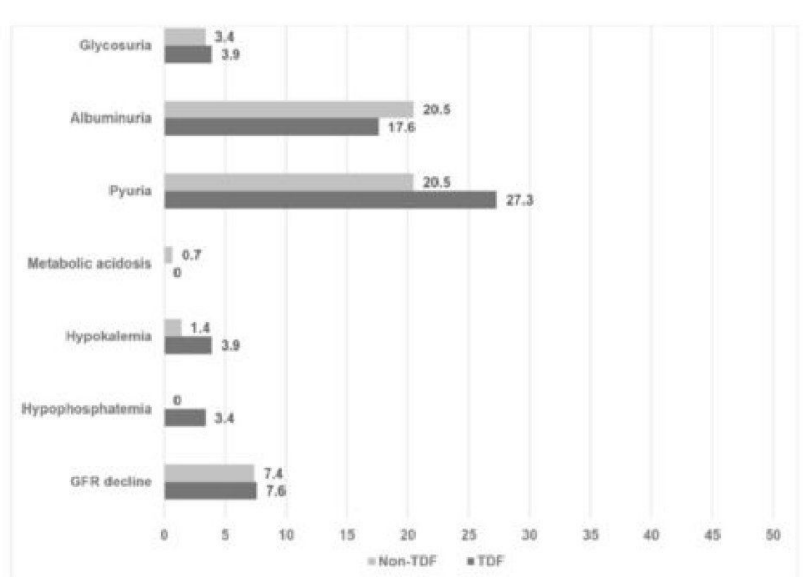

Fig 1. Percentage of renal tubular dysfunction among HIV positive patients receiving TDF $(\mathrm{N}=176)$ and nonTDF regimens $(\mathrm{N}=146)$. All parameters in both groups did not significantly differ $(p>0.05)$. Data are expressed as number (percentage) of patients. Comparisons between treatment groups employed the Chi-square test (Categorical variables). Albuminuria was defined as urine dipstick $>+1$, glucosuria was defined as urine glucose $>+1$, hypophosphatemia was defined as serum phosphorus $<2.5 \mathrm{mg} / \mathrm{dL}$; hypokalemia was defined as serum potassium $<3.5 \mathrm{mg} / \mathrm{dL}$; GFR decline was defined as GFR $<60 \mathrm{~mL} / \mathrm{min} / 173 \mathrm{~m}^{2}$, metabolic acidosis was defined as serum $\mathrm{HCO} 3<18 \mathrm{mEq} / \mathrm{L}$ and pyuria was defined as WBC $<3$ cells/HPF.

\section{Discussion}

This study evaluated the renal safety of TDF among HIV positive patients. Overall, results of this study showed that receiving TDF based antiretroviral therapy exhibited equivalent renal safety as nonTDF based antiretroviral therapy in this patient population. Low frequency of treatment limiting renal impairment and tubular dysfunction was observed among HIV positive patients receiving TDF in Thailand. No statistically significant difference in mean GFR and tubular defect markers was observed between the two groups. Data on the renal safety of TDF in developing countries is limited. Two clinical studies in Caucasian populations reported a low incidence of TDF-associated renal injury and tubular dysfunction. ${ }^{(4,5)}$ Several clinical studies in African populations have also reported approximately 1 to $2 \%$ of TDF-associated nephropathy among HIV positive patients. ${ }^{(6,10,11)}$ One study in a Chinese population showed that patients exposed to TDF regimen exhibited greater renal function decline than the control, but renal function always fluctuated within normal range. ${ }^{(12)}$ Recently, a meta-analysis among 7,496 subjects reported that the risk for acute kidney injury was $0.7 \%$ higher $(95 \% \mathrm{CI}, 0.2$ to 1.2$)$ among TDF-treated subjects than among subjects receiving HARRT without TDF. ${ }^{(13)}$ Similar to our findings, our patients on TDF based antiretroviral therapy were not more likely to experience renal dysfunction than those receiving other regimens.

However, the heterogeneity of renal outcomes with TDF reflected differences in our study design. A high incidence of TDF associated nephropathy have been reported in case control studies or retrospective cohort studies ${ }^{(14)}$, but renal function decline was lower in the studies that systematically reported adverse effects and in randomized clinical trials. ${ }^{(13)}$

Therefore, biochemical laboratory monitoring in terms of long term safety is required regarding randomized control studies. Based on our results and related studies, TDF appeared to be comparable regarding renal safety with other antiretroviral treatments. Concerning normal renal function, TDF-based antiretroviral therapy may be less harmful than previously thought. TDF-induced nephropathy is a reversible form of proximal tubular injury, manifesting distinctive proximal tubular eosinophilic inclusions and ultrastructural mitochondrial abnormalities. ${ }^{(15)}$ Apoptosis and mitochondrial DNA depletion of tubular cells might be involved in the pathogenesis of TDF-induced nephropathy. ${ }^{(16)}$ Clinical manifestation of proximal tubular dysfunction including albuminuria, hypophosphatemia, hypouricemia and tubular acidosis have been described among patients receiving $\operatorname{TDF}^{(9,17,18)}$ Because acid base and electrolyte disturbances are quite common among HIV positive patients and have many possible causes, concluding whether these complications are a direct consequence of TDF is difficult. The etiology of these abnormalities seems to be multifactorial and unrelated to TDF or renal dysfunction. ${ }^{(13,19)}$ In addition, our findings and previous meta-analysis confirm that all proximal tubular dysfunction biomarkers did not differ between TDF-treated and nontreated patients. This study had some limitations. First, because of the crosssectional study, we could not evaluate long term renal outcomes, and we could not assess the mechanisms of TDF associated nephropathy and tubular cell injury. Second, the outcomes of this study were based on primarily Asian patients with normal renal function and may not be generalizable to a special population, e.g., impaired renal function and high comorbid illnesses. Finally, noting that urine electrolytes, uric acid, phosphate and albumin and creatinine ratio were not obtained and measured is important, which may make diagnosis of tubular defects less reliable. 
This study had some limitations. First, because of the cross-sectional study, we could not evaluate long term renal outcomes, and we could not assess the mechanisms of TDF associated nephropathy and tubular cell injury. Second, the outcomes of this study were based on primarily Asian patients with normal renal function and may not be generalizable to a special population, e.g., impaired renal function and high comorbid illnesses. Finally, noting that urine electrolytes, uric acid, phosphate and albumin and creatinine ratio were not obtained and measured is important, which may make diagnosis of tubular defects less reliable.

\section{Conclusion}

In conclusion, among HIV positive patients receiving TDF, mean renal function and electrolyte abnormalities were similar to nonTDF treatment. The authors of this study conclude that TDF-based first line ART can be safely given even without renal monitoring in settings with normal renal function. The renal safety of TDF treatment will require further evaluation in longer duration studies and high risk groups regarding kidney injury.

\section{References}

1. Malik A, Abraham P, Malik N. Acute renal failure and Fanconi syndrome in an AIDS patient on tenofovir treatment-case report and review of literature. J Infect 2005; 51: E61-5.

2. Verhelst $\mathrm{D}$, Monge M, Meynard JL, Fouqueray B, Mougenot B, Girard PM, et al. Fanconi syndrome and renal failure induced by tenofovir: a first case report. Am J Kidney Dis 2002; 40: 1331-3.

3. Laprise C, Baril JG, Dufresne S, Trottier H. Association between tenofovir exposure and reduced kidney function in a cohort of HIV-positive patients: results from 10 years of follow-up. Clin Infect Dis 2013; 56: 567-75.

4. Gallant JE, Parish MA, Keruly JC, Moore RD. Changes in renal function associated with tenofovir disoproxil fumarate treatment, compared with nucleoside reverse-transcriptase inhibitor treatment. Clin Infect Dis 2005; 40: 1194-8.
5. Gallant JE, Winston JA, DeJesus E, Pozniak AL, Chen SS, Cheng AK, et al. The 3-year renal safety of a tenofovir disoproxil fumarate vs. a thymidine analogue-containing regimen in antiretroviral-naive patients. AIDS 2008; 22: 2155-63.

6. Reid A, Stohr W, Walker AS, Williams IG, Kityo $\mathrm{C}$,Hughes $\mathrm{P}$, et al. Severe renal dysfunction and risk factors associated with renal impairment in HIV-infected adults in Africa initiating antiretroviral therapy. Clin Infect Dis 2008; 46: 1271-81.

7. Madeddu G, Bonfanti P, De Socio GV, Carradori S, Grosso C, Marconi P, et al. Tenofovir renal safety in HIV-infected patients: results from the SCOLTA Project. Biomed Pharmacother 2008; 62: 6-11.

8. Nishijima $T$, Komatsu $H$, Gatanaga $H$, Aoki T, Watanabe K, Kinai E, et al. Impact of small body weight on tenofovir-associated renal dysfunction in HIV-infected patients: a retrospective cohort study of Japanese patients. PLoS One 2011; 6: e22661.

9. Gupta SK, Anderson AM, Ebrahimi R, Fralich T,Graham H, Scharen-Guivel V, et al. Fanconi syndrome accompanied by renal function decline with tenofovir disoproxil fumarate: a prospective, case-control study of predictors and resolution in HIV-infected patients. PLoS One 2014; 9: e92717.

10. Stohr W, Reid A, Walker AS, Ssali F, Munderi P,Mambule I, et al. Glomerular dysfunction and associated risk factors over 4-5 years following antiretroviral therapy initiation in Africa. Antivir Ther 2011; 16: 1011-20.

11. Mulenga L, Musonda P, Mwango A, Vinikoor MJ,Davies MA, Mweemba A, et al. Effect of baseline renal function on tenofovir-containing antiretroviral therapy outcomes in Zambia. Clin Infect Dis 2014; 58 : 1473-80.

12. Cao Y, Han Y, Xie J, Cui Q, Zhang L, Li Y, et al. Impact of a tenofovir disoproxil fumarate plus ritonavirboosted protease inhibitor-based regimen on renal function in HIV-infected individuals: a prospective, multicenter study. BMC Infect Dis 2013; 13: 301.

13. Cooper RD, Wiebe N, Smith N, Keiser P, Naicker S, Tonelli M. Systematic review and meta-analysis: renal safety of tenofovir disoproxil fumarate in HIV-infected patients. Clin Infect Dis 2010; 51: 496-505. 
14. Scherzer R, Estrella M, Li Y, Choi AI, Deeks SG, Grunfeld C, et al. Association of tenofovir exposure with kidney disease risk in HIV infection. AIDS 2012; 26: 867-75.

15. Herlitz LC, Mohan S, Stokes MB, Radhakrishnan J, D'Agati VD, Markowitz GS. Tenofovir nephrotoxicity: acute tubular necrosis with distinctive clinical, pathological, and mitochondrial abnormalities. Kidney Int 2010; 78: 1171-7.

16. Perazella MA. Tenofovir-induced kidney disease: an acquired renal tubular mitochondriopathy. Kidney Int 2010; 78: 1060-3.

17. Cheng CY, Chang SY, Lin MH, Ku SY, Sun NL, Cheng SH. Tenofovir disoproxil fumarateassociated hypophosphatemia as determined by fractional excretion of filtered phosphate in HIV-infected patients. J Infect Chemother 2016; 22: 744-7.

18. Haverkort ME, van der Spek BW, Lips P, SliekerWA, ter Heine $\mathrm{R}$, Huitema $\mathrm{AD}$, et al. Tenofovir-induced Fanconi syndrome and osteomalacia in two HIV-infected patients: role of intracellular tenofovir diphosphate levels and review of the literature. Scand J Infect Dis 201 1; 43: 821-6.

19. Day SL, Leake Date HA, Bannister A, Hankins M, Fisher M. Serum hypophosphatemia in tenofovir disoproxil fumarate recipients is multifactorial in origin, questioning the utility of its monitoring in clinical practice. J Acquir Immune Defic Syndr $2005 ; 38: 301-4$. 